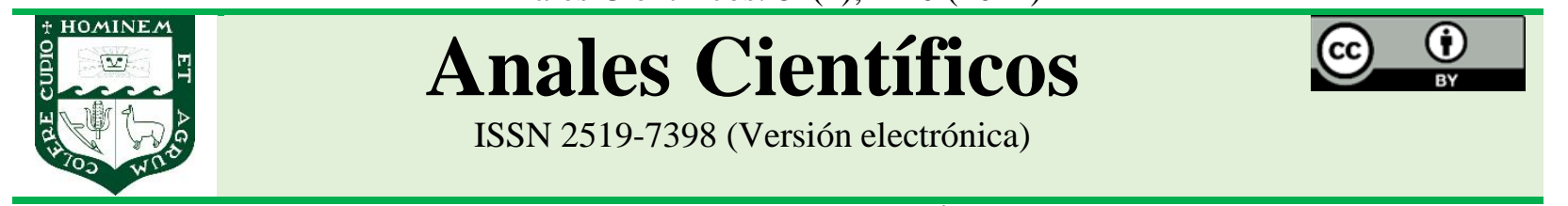

ARTÍCULO ORIGINAL - RESEARCH ARTICLE

http://dx.doi.org/10.21704/ac.v82i1.1736

\title{
GESTIÓN DE LA PROCEDENCIA Y LA TRAZABILIDAD DE PRODUCTOS ORGÁNICOS DE EXPORTACIÓN EN PERÚ
}

\section{Management of origin and traceability of organic export products in Peru}

\author{
Ernesto Altamirano Flores ${ }^{1 *}$; ; Luis Espinoza Villanueva1(i); Luís Raez Guevara² ${ }^{2}$ \\ ${ }^{1}$ Facultad de Economía y Planificación, Universidad Nacional Agraria La Molina, La Molina, 15024, Lima, Perú. \\ ${ }^{2}$ Facultad de Ingeniería Industrial, Universidad Nacional Mayor de San Marcos, 15081, Lima, Perú. \\ * E-mail: ealtamirano@lamolina.edu.pe
}

Recibido: 27/09/2019; Aceptado: 11/02/2021; Publicado: 31/08/2021

\begin{abstract}
The objective of the study was to explain through a model, the influence of chemical compounds in determining the origin and traceability of organic export products, to generate confidence and security in the choice of organic food in consumers, tracing it as purpose the determination of stable isotopes to know the origin and traceability of organic export products, in order to generate confidence and security in the choice of food in consumers. The research methodology was quasi-experimental - descriptive - correlational - explanatory, in addition, data from experiments carried out were used, where the surveys and questionnaires applied in the interview have provided the information that was processed through SPSS program and LISREL for factor analysis. It has been proven that in the studied model the influencing factors originality, traceability and the composition of the food.
\end{abstract}

Keywords: Management; export; organic; marketing and food

\section{RESUMEN}

El estudio tuvo como objetivo, explicar a través de un modelo, la influencia de los compuestos químicos en la determinación de la procedencia y trazabilidad de productos orgánicos de exportación, para generar confianza y seguridad en la elección de alimentos orgánicos en los consumidores, trazándose como propósito la determinación de los isótopos estables para conocer la procedencia y trazabilidad de productos orgánicos de exportación, con la finalidad de generar confianza y seguridad en la elección de alimentos en los consumidores. La metodología de la investigación fue cuasiexperimental - descriptiva- correlacional- explicativo, además se utilizó datos de experimentos realizados, donde las encuestas y los cuestionarios aplicados en la entrevista han brindado la información que fue procesada mediante el programa SPSS y LISREL para el análisis factorial. Se ha comprobado que en el modelo estudiado los factores influyentes son la originalidad, trazabilidad y la composición del alimento.

Palabras clave: gestión; exportación; orgánico; marketing y alimento

Forma de citar el artículo (Formato APA):

Altamirano, E., Espinoza, L., \& Raez, L. (2021). Gestión de la procedencia y la trazabilidad de productos orgánicos de exportación en Perú. Anales Científicos. 82(1), 1-10. http://dx.doi.org/10.21704/ac.v82i1.1736

Autor de correspondencia (*): Ernesto Altamirano Flores. Email: ealtamirano@lamolina.edu.pe (C) Los autores. Publicado por la Universidad Nacional Agraria La Molina. 


\section{INTRODUCCIÓN}

La globalización de mercados agroindustriales y el fácil transporte de alimentos entre países y continentes; las distribuciones de productos etiquetados han incrementado la necesidad de medidas para identificar el origen y trazabilidad de los alimentos orgánicos en el mundo, en este contexto la importancia de las medidas sanitarias y fitosanitarias es indispensable, para ayudar al consumidor en la selección de alimentos (Shalini y Harris, 2013).

Actualmente el análisis de la relación de isótopos estables, en combinación con otros métodos químicos se convierte en un enfoque prometedor para determinar la autenticidad y la trazabilidad, en exportaciones de alimentos orgánicos.

En los últimos cinco años, un creciente número de trabajos de investigación han sido publicados en el rastreo de los productos orgánicos por relación de isótopos estables, análisis y técnicas que combinan con otros instrumentos (Zhao y Zhang, 2013).

La procedencia de subproducto orgánico es de particular importancia para la protección de los consumidores; el origen geográfico es otro factor esencial para la evaluación de la calidad de alimentos agroindustriales, para no causar cambios en el valor nutricional, pero en términos de engaño al consumidor, la venta de productos extranjeros baratos como de alto precio agro-producto regional. El subproducto agroindustrial falsificado y la utilización falsa de la indicación geográfica son perjudiciales para los consumidores y los productores legítimos (Zhao y Zhang, 2013).

La transformación en la composición de las exportaciones agroalimentarias de los países en desarrollo, con una rápida expansión de las exportaciones de productos no tradicionales, ha servido para tomar conciencia en el consumo de productos etiquetados de confianza, la seguridad alimentaria en vegetales y animales con medida sanitaria y fitosanitarias (Shalini y Harris, 2013).
Casi todos los elementos químicos conocidos (carbono, oxígeno, hidrógeno, nitrógeno, etcétera) existen en varias formas que difieren en el número de neutrones en su núcleo, a estas formas se las llama isótopos. Algunas de estas formas son inestables y sufren decaimiento radioactivo (Sánchez, 2009).

La cantidad de nutrientes es similar en los alimentos de producción convencional y ecológica. En los vegetales, el valor alimenticio es similar. En las carnes, está comprobado que las orgánicas son más magras porque tienen menos grasa intramuscular (Duran, 2010).

Los procesos de recopilación, procesamiento y análisis de información, respecto a temas relacionados con la mercadotecnia, como: clientes, competidores y el mercado son indispensables en esta investigación (Muriel et al., 2015).

La investigación tiene como problema principal ¿Cuál es la influencia de los compuestos químicos de los alimentos en la determinación de la autenticidad y trazabilidad de productos orgánicos de exportación, para generar confianza y seguridad en la elección de alimentos orgánicos en los consumidores?

El objetivo de la investigación fue determinar la influencia de los compuestos químicos en la procedencia y trazabilidad de productos orgánicos de exportación, con la finalidad de generar confianza y seguridad en la elección de alimentos en los consumidores.

\section{MATERIALES Y MÉTODOS}

\section{Materiales y tipo de investigación}

Se utilizó una Laptop, un USB, un sofware SSPS y hojas de práctica. La investigación fue cuasiexperimental - correlacional- explicativo, se realizó con datos de experimentos realizados, donde las encuestas y cuestionarios de la entrevista proveen la información para procesar mediante programas de SPSS y análisis factorial. 
Se estudiaron grupos intactos, los grupos fueron seleccionados al azar. No se da una equiprobabilidad de los sujetos en los grupos experimental y de control. Pero se mantiene un control parcial aceptable, por manipular al menos una variable independiente.

El alcance de la investigación es Investigación explicativo, porque se ocupa de la descripción de datos características de una población, asimismo por que raramente involucran experimentación, ya que están más preocupados con los fenómenos que ocurren naturalmente que con la observación de situaciones controladas.

Mientras tanto, investigación correlacional, porque consiste en la búsqueda de algún tipo de relación entre dos o más variables, y en qué medida la variación de una de las variables afecta a la otra, sin llegar a conocer cuál de ellas puede ser causa o efecto.

El enfoque de la investigación es cuantitativo y cualitativo, cuantitativa por que el procedimiento de decisión que pretende señalar, entre ciertas alternativas, son usando magnitudes numéricas que pueden ser tratadas mediante herramientas del campo de la estadística, cualitativa por considerar técnicas cualitativas distintas a la encuesta y al experimento.

\section{Unidad de análisis}

Son las personas que participan en la compra diaria, integrantes de familia o empleadas del hogar que eligen los alimentos en el supermercado.

\section{Población}

La población está representada por los consumidores de cuatro supermercados más importantes de Alemania, personas que participan en la compra constante de los principales alimentos de exportación de productos orgánicos.

\section{Tamaño de muestra}

El tamaño de la muestra son las personas de 18 a 60 años de edad que participan en la compra y elección de alimentos orgánicos importados para el consumo diario, que representan 100 personas de Alemania.

\section{Selección de muestra}

La selección de muestra se ha determinado según la fórmula de muestreo proporcionada por Mayorga \& Araujo (2010), cuando la población es finita, los cálculos y parámetros considerados se presentan a continuación.

$$
n=\frac{Z^{2} \cdot p \cdot q \cdot N}{e^{2} \cdot(N-1)+Z^{2} \cdot p \cdot q}
$$

Dónde:

$$
\begin{aligned}
& \mathrm{n}=\text { muestra } \\
& \mathrm{N}=\text { Población } 100 \text { consumidores } \\
& \mathrm{Z} 2=\text { Nivel de confianza }(95 \%-1,96) \\
& \mathrm{e} 2=\text { Error permitido }(5 \%) \text { ó }(0,05) \\
& \mathrm{p}=\text { Proporción de que el evento ocurra en un } 0,5(50 \%) \\
& \mathrm{q}=\text { Proporción de que el evento no ocurra en un } 0,5(50 \%) \\
& \mathrm{n}=34,5744 / 1,0479=79,5 \text { Por lo tanto, se encuestó } 80 \\
& \text { consumidores }
\end{aligned}
$$

El objetivo de las encuestas es recabar información primaria, para diseñar un modelo para la generación de confianza en la selección de alimentos orgánicos de exportación (Figura 1).

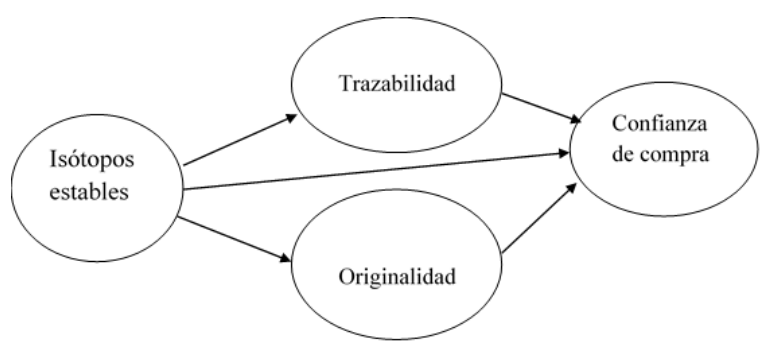

Figura 1. Modelo para la generación de confianza en la selección de alimentos orgánicos de exportación

\section{Técnica de recolección de datos}

Se realizó una encuesta para la obtención de información sobre el estado actual de los consumidores de productos orgánicos importados. La encuesta se realizó después de seleccionar cuatro alimentos (carne, vino, leche y aceite) más consumidos de productos orgánicos importados en Alemania, luego se realizó 30 preguntas relacionadas al producto orgánico y la relación de isótopos estables, con esta información se desarrolló un modelo para determinar cuánto influye estos métodos químicos en la gestión de compra.

La entrevista se realizó mediante el empleo una guía de entrevistas tipo cuestionario a consumidores y 
participantes en la compra de productos orgánicos importados, que constituye un documento o formato escrito de preguntas relacionadas con el objetivo del estudio.

\section{Análisis e interpretación de la información}

La clasificación de datos, registro y codificación de datos, se realizó con la selección de respuestas a las encuestas y cuestionarios para los tres puntos determinantes en la investigación, como: composición de alimentos orgánicos, selección de alimentos orgánicos y satisfacción del cliente, estas informaciones se codifica de acuerdo a las respuestas cuantificables, es base para los análisis cuantitativos donde se utilizó estadísticos descriptivos de tendencia central y para lo cualitativo la Razón.

Las técnicas analíticas estadísticas que se utilizó para comprobar los resultados y obtener las conclusiones, fueron los análisis estadísticos de varianza de los datos de la investigación extraídos de las encuestas a través de las preguntas del cuestionario, procesados con programas de SPSS. 20 y LISREL para el diseño factorial.

\section{RESULTADOS Y DISCUSIÓN}

En la investigación sobre la gestión de la originalidad y trazabilidad de alimento de exportación, se obtuvieron resultados de los alimentos más comercializados a nivel internacional, específicamente en el mercado europeo, donde a partir de la literatura y la aplicación de modelos los resultados, por ejemplo, para uno de los alimentos de mayor desconfianza $(13 \mathrm{C}, 15 \mathrm{~N}, 18 \mathrm{O}, 2 \mathrm{H}, 34 \mathrm{~S})$ demostraron ser susceptibles al análisis de isótopos, por el cual diversos grados de éxito fue lograda. Actualmente existe literatura sobre el uso de análisis de isótopos estables, junto con otros análisis de las técnicas pertinentes de los productos de origen animal autenticidad y trazabilidad) (Zhao y Zhang, 2013).

Trazabilidad geográfica, carne de vacuno mediante isótopos estables, en primer lugar, comenzó en Europa, centrándose inicialmente en el análisis de la composición de isótopos de un solo elemento, luego se extendió a la combinación de múltiples elementos (Zhao y Zhang, 2013).

El examen de la relación isotópica estable utilizada fueron el oxígeno y el hidrógeno para caracterizar el origen geográfico del ganado.

Agua meteórica que ha pasado a través de la meteorología ciclo de evaporación, condensación y precipitación finalmente constituye una de las aguas subterráneas que exhibe una sistemática variación isotópica geográfica (Zhao y Zhang, 2013).

La variación global en sistemática $2 \mathrm{H}$ y $18 \mathrm{O}$ contenido de la precipitación se transfiere a través agua o alimentación potable en carne. Se ha demostrado por que el valor único \%o d18O de agua de los tejidos puede diferenciar el origen de la carne de vacuno procedentes de Alemania, Argentina y Reino Unido, en el que las muestras de carne de vacuno procedentes de Alemania y Argentina puede ser claramente clasificado detectando el valor $\mathrm{d} 18 \mathrm{O}$ $\%$ de agua de los tejidos, mientras que el valor $\mathrm{d} 18 \mathrm{O}$ $\%$ no proporciona suficiente resolución para discriminar las muestras de Reino Unido de los de alemán y Argentina. Tampoco se permite la discriminación clara entre la región norte y sur de la producción dentro de Alemania (Zhao y Zhang, 2013).

Para diferenciar las muestras de carne a partir de tres granjas en Alemania, se aplicó la adición de $\mathrm{N}$ y S como composición. Sobre la base de los resultados anteriores, el análisis de $\mathrm{D} / \mathrm{H}$ y las proporciones de 18O/16O del agua de los tejidos pueden ser considerados como un potencial muy herramienta para la identificación del origen de la carne (Zhao y Zhang, 2013).

En otro aspecto, sin embargo, la influencia del tiempo de almacenamiento sobre la composición de isótopos de oxígeno de agua de los tejidos fue investigado por lo que indica que se observó $2 \%$ variación por el valor de $\mathrm{d} 18 \mathrm{O}$ después de $24 \mathrm{~h}$ de almacenamiento de la carne a los $0{ }^{\circ} \mathrm{C}$. También se ha encontrado que el valor de $\mathrm{d} 18 \mathrm{O}$ del jugo de carne podría ser posiblemente cambiado durante el proceso de tostado. Como resultado, el análisis d180 de agua de los tejidos puede ser limitada en la 
asignación origen geográfico ya que los valores d180 pueden verse afectados por diversas condiciones de procesamiento y almacenamiento. Por consiguiente, la medición de la relación isotópica estable entre hidrógeno y oxígeno se debe evitar el riesgo (Zhao y Zhang, 2013).

En comparación con el análisis de la composición de isótopos solo elemento para averiguar el origen geográfico de la carne, el análisis de múltiples composiciones de isótopos estables de masa seca desgrasada es el método más potente. Multielemento $(\mathrm{H}, \mathrm{C}, \mathrm{N}, \mathrm{S})$ proporciones de isótopos estables en desgrasada masa seca se han detectado, para trazar el cordero orígenes geográficos de las 13 regiones europeas (Zhao y Zhang, 2013).

El carbono relación isotópica en productos de origen animal se basó en la planta C3 y C4 en la dieta de los animales. Un mayor contenido de $13 \mathrm{C}$ en materia seca desgrasada es un indicador de C4-planta de maíz alimentado animal. En otro aspecto, el contenido de deterioro de proteínas secas para el cordero está en buen acuerdo con que en el agua la precipitación y la tierra. Usando este método, $78 \%$ de clasificación correcta se puede lograr en base a multivariante análisis estadístico (Zhao y Zhang, 2013).

En un rango mayor, las muestras de carne se compararon con las principales regiones del mundo productoras de ganado (incluyendo Europa, EEUU., América del Sur, Australia y Nueva Zelanda). Al detectar las composiciones isotópicas de $\mathrm{C}$ y $\mathrm{N}$ de masa seca desgrasada y $\mathrm{H}$ y composiciones $\mathrm{O}$ isótopos de lípidos, se ha encontrado que el contenido de $13 \mathrm{C}$ de carne de vacuno producida en Brasil y EE.UU. fue significativamente mayor que el de la carne de vacuno británica, ya que el maíz intensivo y pastos $\mathrm{C} 4$ eran alimentados al ganado en Brasil y EE.UU., mientras que en Gran Bretaña C3 pastos y forraje dominada (Zhao y Zhang, 2013).

Los valores \% D2H media y d180 \%o de lípidos carne estaba bien correlacionada con la latitud de la región de producción, y la relación entre el $\mathrm{H}$ y O contenido isotópico se encontró que era paralela a la línea de agua meteórica. Multi-elemento $(\mathrm{C}, \mathrm{N}, \mathrm{H}$, S) también se utilizó el análisis de isótopos de carne muscular como una analítica herramienta para la verificación de los orígenes geográficos incluidos varios países europeos y no europeos (Zhao y Zhang, 2013).

Los valores isotópicos de medias para los diferentes países eran de diferencia altamente significativa entre sí, y 84,9\% de las muestras se podrían asignar correctamente por el análisis (Zhao y Zhang, 2013).

Además de los estudios de trazabilidad geográficas en Europa y los Estados Unidos, hay también investigaciones relacionadas que se han llevado a cabo por científicos de países asiáticos como Japón, Corea y China en el pasado cinco años, incluyendo la diferenciación de la carne local desde el importado producto y la clasificación de los productos cárnicos nacionales desde diferentes ubicaciones (Zhao y Zhang, 2013).

En la investigación se encontró que, el carbono y nitrógeno isotópica parcelas composición para muestras de carne de vacuno japoneses, estaban en un rango estrecho, por su parte, la composición isotópica de oxígeno de proteína desgrasada se indica una correlación positiva con la de agua local.

\section{Arroz}

El origen geográfico del arroz puede ser identificado por $87 \mathrm{Sr} / 86 \mathrm{Sr}$. La relación $87 \mathrm{Sr} / 86 \mathrm{Sr}$ de arroz de China y Vietnam oscilaron $0,710-0,711$, mientras que la más alta relación $87 \mathrm{Sr} / 86 \mathrm{Sr}$ se observó en el intervalo de 0,715 a 0,717 para el arroz de Australia, lo que indica que el estroncio isótopos estables podría ser un indicador único para la estimación de la procedencia de arroz (Zhao y Zhang, 2013).

La investigación se realizó mediante la combinación de estroncio y proporciones de isótopos de boro. Arroz muestras, recogidas en Japón, EE.UU., China, Australia, Corea y Vietnam, podrían distinguirse por solo estroncio composición isotópica solo o junto con la relación de 11B / 10B. Como resultado, el estroncio f puede ser útil para la determinación de la autenticidad, debido a su diferente alcance de los elementos ligeros. El estroncio tiene cuatro isótopos naturales: $84 \mathrm{Sr}, 86 \mathrm{Sr}, 87 \mathrm{Sr}$ y $88 \mathrm{Sr}$ (Kelly y Heaton, 2005). 
Aunque los procesos biológicos pueden alterar de estroncio de isótopos estables ratios en una manera similar como con los isótopos $\mathrm{S}, \mathrm{C}, \mathrm{H}, \mathrm{O}$ y $\mathrm{N}$, solamente la cantidad de exceso radiogénico $87 \mathrm{Sr}$ se evalúa como un trazador de origen.

Para rastrear los cultivos de cereales por los isótopos estables de los elementos ligeros, una variedad de elementos (d13C, d15N, d2h y d18O) han sido investigados.

Muestras de arroz recogidas en los EE.UU., Europa, India y Pakistán se analizaron los isótopos estables y elementos minerales (Kelly y Heaton, 2005), lo que indica que las muestras de arroz de la India y Pakistán se caracterizan por su valor más bajo en comparación $18^{\circ}$ a las muestras de Estados Unidos y Europa. Nueve parámetros (d13C, d18O, boro, holmio, gadolinio, magnesio, rubidio, selenio y tungsteno) fueron analizados para identificar el origen geográfico de análisis discriminante canónico por etapas, que mostró la discriminación $100 \%$ entre las muestras de arroz de diferentes orígenes.

La natural abundancia de relaciones isotópicas de $\mathrm{C}$, $\mathrm{O}$ y $\mathrm{N}$ fueron comparados entre las muestras de trigo procedentes de Italia, Canadá, Turquía y Australia para su contenido isotópico (Brescia et al., 2002).

\section{Vino}

Debido al precio relativamente más alto de vino en comparación con otros productos agrícolas, hay un gran número de informes utilizando análisis isotópico para la identificación de la procedencia de los vinos. Inicialmente, 180 / 160 se desempeñó como un parámetro clave para confirmar la adición de agua y falsa declaración del origen de los vinos (Zhao y Zhang, 2013).

\section{Aceite}

Una estrategia analítica eficaz para verificar la autenticidad de aceite puede ser proporcionada por análisis de la relación de isótopos estables junto con otras técnicas. La combinación de FINE-RMN y IRMS era utilizado para discriminar el aceite de mostaza auténtica del fraude aceite con isotiocianato de alilo sintético añadido (Zhao y Zhang, 2013).
El método permitió una clara distinción entre lo natural y adulteración de mostaza natural aceite mediante el análisis de los parámetros isotópicos incluidos 13C / 12C, $15 \mathrm{~N} / 14 \mathrm{~N}, 34 \mathrm{~S} / 32 \mathrm{~S}$ y $2 \mathrm{H} /$ 1H. Análisis de isótopos estables fue para diferenciar el origen de escualeno y escualano su hidrogenado, en el que la capacidad de d13C y d2h fue investigado por distinguir el aceite de ambas fuentes: aceite de hígado de aguas profundas tiburones y destilados de aceite de oliva (Zhao y Zhang, 2013).

\section{Fiabilidad de todas las variables}

Sobre las 80 encuestas con 30 preguntas (cada una es una variable). Los comandos en SPSS para calcular el Alfa de Cronbach son: Analizar/Escala/ Análisis de fiabilidad/ Estadístico/Escala; si se elimina el elemento/ correlaciones (Tabla 1).

Tabla 1. Resumen de procesamiento de casos.

\begin{tabular}{llcc}
\hline & & $\mathbf{N}$ & $\mathbf{\%}$ \\
\hline \multirow{2}{*}{ Casos } & Válido & 80 & 100,0 \\
& Excluido $^{\text {a }}$ & 0 & 0 \\
\hline \multirow{2}{*}{ Total } & 80 & 100,0 \\
\hline
\end{tabular}

a. La eliminación por lista se basa en todas las variables del procedimiento.

A través de la aplicación estadística del SPSS, el resultado de Alfa de Cronbach se obtiene 0,822 (Tabla 2).

Tabla 2. Estadísticas de fiabilidad.

\begin{tabular}{ccc}
\hline $\begin{array}{c}\text { Alfa de } \\
\text { Cronbach }\end{array}$ & $\begin{array}{c}\text { Alfa de Cronbach } \\
\text { basada en elementos } \\
\text { estandarizados }\end{array}$ & $\begin{array}{c}\text { N de } \\
\text { elementos }\end{array}$ \\
\hline 0,815 & 0,822 & 30 \\
\hline
\end{tabular}

El valor de Alfa de Cronbach de 0,822 es aceptable para poder confiar en los datos obtenidos. 


\section{Herramienta de análisis para la contrastación de hipótesis}

Las respuestas de las encuestas se analizaron mediante pruebas de significación (chi cuadrado y pruebas) en SPSS 20 y el modelo se determinó con software LISREL.

En análisis e interpretación de la información se usó la herramienta de distribución normal o la prueba de normalidad, para el análisis descriptivo y contrastación de hipótesis (Tabla 3).

\section{Prueba de normalidad}

Se efectuaron las pruebas de normalidad, habiendo obtenido resultados de significancia, para buena y muy buena (Tabla 3).

Tabla 3. Pruebas de normalidad.

\begin{tabular}{llcccccc}
\hline VD & & \multicolumn{2}{c}{ Kolmogorov-Smirnov $^{\mathbf{a}}$} & \multicolumn{3}{c}{ Shapiro-Wilk $^{\mathbf{b}}$} \\
(agrupado) & & Estadístico & gl & Sig. & Estadístico $^{\text {gl }}$ & Sig. \\
\hline VI & Buena & 0,256 & 7 & 0,182 & 0,833 & 7 & 0,004 \\
(agrupado) & Muy buena & 0,403 & 69 & 0,000 & 0,654 & 69 & 0,000 \\
\hline
\end{tabular}

a. Corrección de significación de Lilliefors

b. VI (agrupado) es constante cuando VD (agrupado) = Excelente. Se ha omitido.

Interpretación: Como se puede observar en la Tabla 4 estadístico, el $\mathrm{P}$ valor es menor al nivel de significancia, por lo que concluye que los datos no son distribuidos como una normal, es decir, los datos no son normales. Por lo tanto, el método que se va a usar es el Spearman.
Análisis de la prueba de correlación de la hipótesis Isótopos relacionados con originalidad, trazabilidad y confianza en la elección de alimentos, la correlación de hipótesis (Tabla 4).

Tabla 4. Correlaciones.

\begin{tabular}{|c|c|c|c|c|}
\hline & & & $\begin{array}{c}\text { VI } \\
\text { (agrupado) }\end{array}$ & $\begin{array}{c}\text { VD } \\
\text { (agrupado) }\end{array}$ \\
\hline \multirow{6}{*}{$\begin{array}{l}\text { Rho de } \\
\text { Spearman }\end{array}$} & \multirow[t]{3}{*}{ VI (agrupado) } & Coeficiente de correlación & 1,000 & $0,490^{* *}$ \\
\hline & & Sig. (bilateral) & . & 0,000 \\
\hline & & $\mathrm{N}$ & 80 & 80 \\
\hline & \multirow{3}{*}{$\begin{array}{l}\text { VD } \\
\text { (agrupado) }\end{array}$} & Coeficiente de correlación & $0,490^{* *}$ & 1,000 \\
\hline & & Sig. (bilateral) & 0,000 & . \\
\hline & & $\mathrm{N}$ & 80 & 80 \\
\hline
\end{tabular}

** La correlaciónes significativa en el nivel 0,01 (2 colas).

La variable originalidad está influido por la respuesta que corresponde a la pregunta ¿Considera que los alimentos orgánicos son más saludables de manera, y la ¿Cómo considera en la composición de alimentos de los países sudamericanos?, donde se determinó el alto valor de importancia para su toma de decisión en la compra de productos alimenticios (Figura 2).

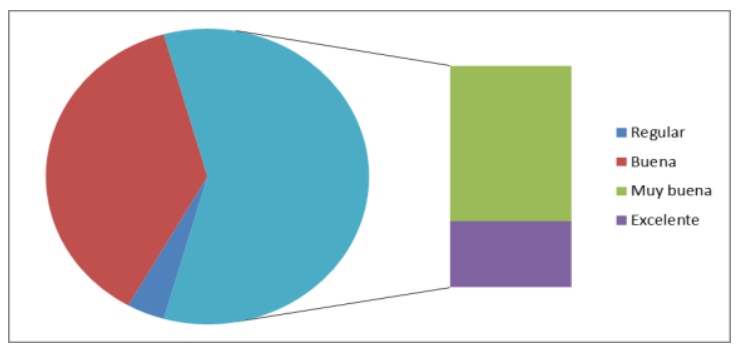

Figura 2. Consideraciones de los alimentos orgánicos son más saludables. 
La consideración en la composición de alimentos de los países sudamericanos también fue un factor determinante para el diseño del modelo (Figura 3).

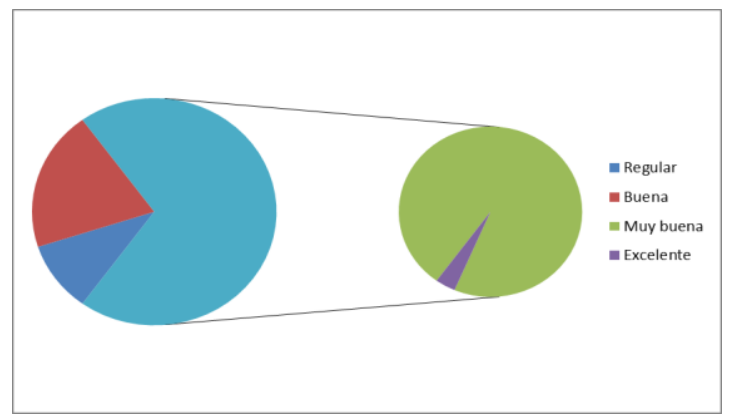

Figura 3. Consideraciones de la composición de alimentos de los países sudamericanos.

La variable isótopos estables es un factor importante en el modelo a partir de la influencia de las preguntas ¿Considera que los alimentos orgánicos son más saludables de manera? Considera importante el contenido de la composición de los alimentos orgánicos importados que consume. ¿Cómo considera la comida orgánica para su elección de alimentos? El resultado es isótopos estables = $2,07 * \mathrm{v} 4+1,09 * \mathrm{v} 1+1,0 * \mathrm{v} 5+0,07$.

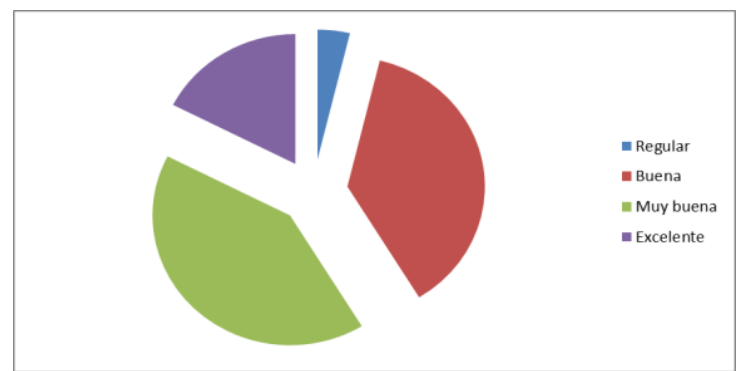

Figura 4. Consideraciones de la comida orgánica para su elección de alimentos.

La variable de importancia para este diseño del modelo también es el resultado de la ¿Cómo considera en la composición de alimentos de los países sudamericanos?

$\mathrm{La}$ variable trazabilidad es igual a $6,46 * \mathrm{v} 21+6,14 * \mathrm{v} 20+5,46 * \mathrm{v} 16+5,30 * \mathrm{v} 15+0,02$, que es resultado de las preguntas ¿Qué percepción tienen en los productos orgánicos no procesados? ¿Qué percepción tienen en los productos orgánicos procesados? ¿Cómo considera el proceso de producción de productos orgánicos de otros países? ¿Qué percepción tiene de la comercialización de productos orgánicos en el mercado de Alemania? La pregunta ¿Cómo considera el grado de confianza en la elección de alimentos extranjero? Influye en la confianza $* 0,53+$ trazabilidad $* 2,61+3,02$.

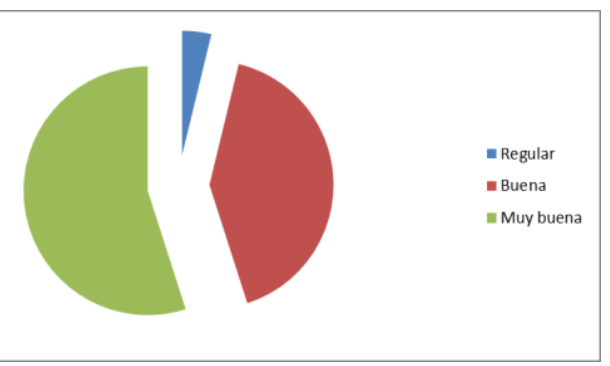

Figura 5. Consideraciones en la composición de alimentos de los países sudamericanos.

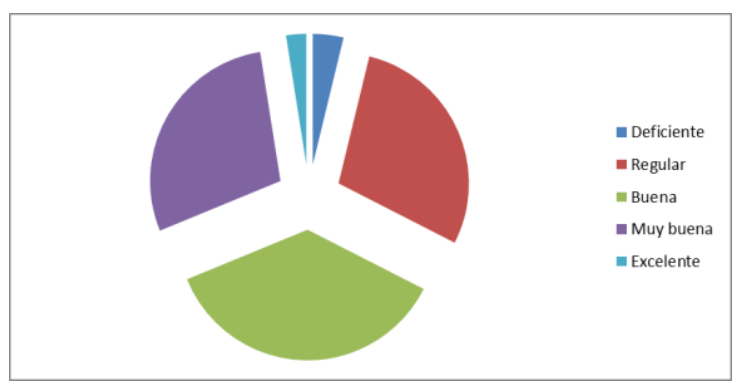

Figura 6. Percepción sobre productos orgánicos no procesados.

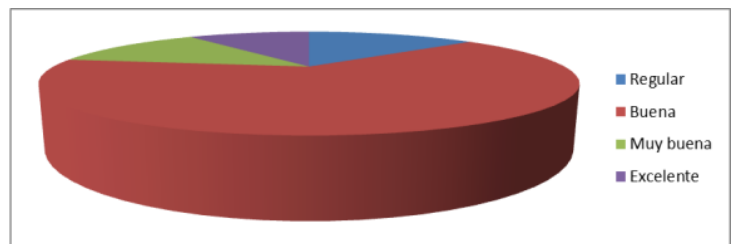

Figura 7. Consideraciones al grado de confianza en la elección de alimento extranjero.

Hempel y Hamm (2016), en su investigación, la importancia de la comida local determinó que la autenticidad de los subproductos agroalimentarios y la trazabilidad es la principal preocupación no sola de los consumidores, también lo es para los agricultores agrícolas, los minoristas y las autoridades. 
El estudio se ocupa de las actitudes de los consumidores alemanes hacia los alimentos orgánicos y comida local, su comida, comportamiento de compra y sus características personales.

El propósito es investigar las diferencias en actitudes y valores de la disposición a pagar entre los consumidores que consideran que la producción orgánica de alimentos importante y quienes lo consideran menos importantes, donde los resultados obtenidos tienen diferencias estadísticas en resultados de la percepción de los consumidores europeos y los sudamericanos, esto se puede observar son la comparación por ejemplo del $90 \%$ de los europeos les importa la trazabilidad de un alimento, es diferente en relación a los resultados obtenidos en esta investigación donde solo $67 \%$ de sudamericanos importa la procedencia y trazabilidad del alimento, entonces la investigación anterior algunos resultados no nos similares, pero esto explica la cultura de las personas en cada continente (Jones y Davidson, 2014). La adaptación a las crisis de seguridad alimentaria: Interpretación de éxito y el fracaso en la respuesta de Canadá a la EEB, explora los procesos de adaptación a las crisis de seguridad alimentaria, y plantea interrogantes acerca de lo que puede ser entendida como el éxito y el fracaso en una respuesta a la crisis. Presenta los resultados de una investigación cualitativa estudio de la industria de la carne asediado de Canadá e investiga el aprendizaje institucional y la adaptación siguiente un brote de EEB (encefalopatía espongiforme bovina), en relación a esta investigación el modelo actual tiene respuesta rápida, por la determinación del compuesto se puede determinar las molécula de los microrganismos y también evitar el terrorismo biológico, entonces es una metodología que aporta mayor eficiencia y eficacia a la decisión de compra.

El análisis y la configuración del modelo arroja que la confianza del comprador es resultado de originalidad, trazabilidad y la composición de los isótopos estables, como sigue: confianza es 10,34 impacto del diseño del modelo, la originalidad es otro factor de impacto más importante para el modelo estructurado con 1,59 de impacto, los isótopos estables con un 20,92 y la trazabilidad con un 0,22 de impacto el menos influyente para el diseño estructural de modelo.

\section{CONCLUSIONES}

Del estudio se concluye que, para determinar la influencia del origen y trazabilidad en la selección de alimentos en Europa, se comprueba mediante un modelo que los factores influyentes son la originalidad, trazabilidad y la composición del alimento, esto analizando respuestas de los compradores en la encuesta. Además, el modelo utilizado en la investigación es efectivo para determinar resultados de la composición como la carne con un valor $13 \mathrm{C} / 12 \mathrm{C}$ porque el maíz se utiliza como alimentación convencional. El vino en comparación con un valor de 180 / 160, el arroz con un valor de $87 \mathrm{Sr} / 86 \mathrm{Sr}$. La relación $87 \mathrm{Sr} / 86 \mathrm{Sr}$ de arroz de China y El aceite con un valor de 13C / 12C, $15 \mathrm{~N} / 14 \mathrm{~N}, 34 \mathrm{~S} / 32 \mathrm{~S}$ y $2 \mathrm{H} / 1 \mathrm{H}$, confirman la diferencia de composición de acuerdo con el lugar de origen trazabilidad. Asimismo, la diferencia de cultura europea y Sudamérica influye en la toma de decisiones a la hora de comprar alimentos, existe mayor preocupación en el cliente de la Unión Europea en la trazabilidad de productos donde el $90 \%$ es consciente de la composición del producto y en Sudamérica solo 67\%. Se recomienda investigar otros factores que puedan influir en el modelo, estas investigaciones pueden ayudar determinar el origen geografía de alimentos importado en Sudamérica con mayor efectividad, en específico en el Perú

\section{Conflictos de intereses}

Los autores firmantes del presente trabajo de investigación declaran no tener ningún potencial conflicto de interés personal o económico con otras personas $\mathrm{u}$ organizaciones que puedan influir indebidamente con el presente manuscrito.

\section{Contribuciones de los autores}

Preparación y ejecución: EA, LE, LR; Desarrollo de la metodología: EA, LE, LR; Concepción y diseño: EA, LE, LR; Edición del artículo: EA, LE, LR; Supervisión del estudio: EA, LE, LR. 


\section{LITERATURA CITADA}

- Duran, O. (2010). Alimentarse con orgánicos, convencionales o transgénicos en Costa Rica. ISSN 1409-214X. Ambientico 242, Artículo 1 Pp. 4-11.

- Hempel, C., \& Hamm, U. (2015). ¿Qué tan importante es la comida local a los consumidores orgánicos mente? Importancia de la alimentación sana. Alemania. Journal Homepage, Scopus, 7 pp.

- Jones y Davidson, (2014). La evolución de desempeño agroambiental de las explotaciones lecheras en el noreste de Italia se basa en resultados agregados de los indicadores. Journal Homepage Scopus, 8 pp.

- Kelly, S., Heaton, K., \& Hoogewerff, J. (2005). Tracing the geographical origin of food: The application of multi-element and analysis. Trends in Food Science \& Technology, 16, 555567. https://doi.org/10.1016/j.tifs.2005.08.008.
- Mayorga, D., \& Araujo, P. (2010). El plan de Marketing. Centro de investigación. Universidad del Pacifico. Lima, Perú. 134 pp.

- Muriel, C., \& Siet, J. (2015). La segmentación de los consumidores basado en la categoría de los alimentos atributo importante: la relación con la salubridad y sostenibilidad percepciones. UNMSM, Lima-Perú.

- Sánchez, L. (2011). Isótopos Estables de Oxígeno y Carbono en Foraminíferos del Margen Continental de Magdalena, B.C.S. México.

- Shalini, A., \& Harris, N. (2013). La evaluación de la pertenencia de las medidas sanitarias y fitosanitarias de la UE en el sector de las exportaciones de alimentos: la evidencia de un país exportador agroalimentario en desarrollo. Journal Homepage, Scopus, 13 pp.

- Zhao, Y., \& Zhang, B. (2013). Acontecimientos recientes en la aplicación de análisis de isótopos estables en la autenticidad de agro productos y trazabilidad. Journal Homepage, Scopus, 5 pp. 